\title{
Prediction of serosal invasion in gastric cancer: development and validation of multivariate models integrating preoperative clinicopathological features and radiographic findings based on late arterial phase $\mathrm{CT}$ images
}

Song Liu ${ }^{1+}$, Mengying $\mathrm{Xu}^{1+}$, Xiangmei Qiao ${ }^{1+}$, Changfeng $\mathrm{Ji}^{1}$, Lin $\mathrm{Li}^{2^{*}}$ and Zhengyang Zhou ${ }^{{ }^{*}}$

\begin{abstract}
Background: To develop and validate multivariate models integrating endoscopic biopsy, tumor markers, and CT findings based on late arterial phase (LAP) to predict serosal invasion in gastric cancer (GC).

Methods: The preoperative differentiation degree, tumor markers, CT morphological characteristics, and CT valuerelated and texture parameters of 154 patients with GC were analyzed retrospectively. Multivariate models based on regression analysis and machine learning algorithms were performed to improve the diagnostic efficacy.

Results: The differentiation degree, carbohydrate antigen (CA) 199, CA724, CA242, and multiple CT findings based on LAP differed significantly between T1-3 and T4 GCs in the primary cohort (all $P<0.05$ ). Multivariate models based on regression analysis and random forest achieved AUCs of 0.849 and 0.865 in the primary cohort, respectively.

Conclusion: We developed and validated multivariate models integrating endoscopic biopsy, tumor markers, CT morphological characteristics, and CT value-related and texture parameters to predict serosal invasion in GCs and achieved favorable performance.
\end{abstract}

Keywords: Stomach neoplasms, Neoplasm staging, Tomography, X-ray computed, Endoscopy, Biomarkers, tumor

\footnotetext{
* Correspondence: lilinpathology@163.com; zyzhou@nju.edu.cn

${ }^{\dagger}$ Song Liu, Mengying Xu and Xiangmei Qiao contributed equally to this work.

${ }^{2}$ Department of Pathology, Nanjing Drum Tower Hospital, The Affiliated Hospital of Nanjing University Medical School, Nanjing 210008, China 'Department of Radiology, Nanjing Drum Tower Hospital, The Affiliated Hospital of Nanjing University Medical School, No.321, Zhongshan Road, Nanjing City 210008, Jiangsu Province, China
}

C C The Author(s). 2021 Open Access This article is licensed under a Creative Commons Attribution 4.0 International License, which permits use, sharing, adaptation, distribution and reproduction in any medium or format, as long as you give appropriate credit to the original author(s) and the source, provide a link to the Creative Commons licence, and indicate if changes were made. The images or other third party material in this article are included in the article's Creative Commons licence, unless indicated otherwise in a credit line to the material. If material is not included in the article's Creative Commons licence and your intended use is not permitted by statutory regulation or exceeds the permitted use, you will need to obtain permission directly from the copyright holder. To view a copy of this licence, visit http://creativecommons.org/licenses/by/4.0/ The Creative Commons Public Domain Dedication waiver (http://creativecommons.org/publicdomain/zero/1.0/) applies to the data made available in this article, unless otherwise stated in a credit line to the data. 


\section{Background}

Gastric cancer (GC) is the fifth most common cancer and the third leading cause of cancer-related deaths globally and has become one of the major health burdens [1]. Previous studies have confirmed that serosal invasion is closely related to peritoneal seeding, which is generally regarded as a terrible condition [2, 3]. Thus, the precise preoperative prediction of serosal (visceral peritoneum) invasion is vital to select appropriate treatments and predict the outcome of GC. For instance, treatments such as staging laparoscopy and neoadjuvant chemotherapy would be scheduled in more detail if serosal (visceral peritoneum) invasion occurs [4, 5].

Both the seventh and eighth editions of the American Joint Cancer Committee divided the GC stage of T4 into $\mathrm{T} 4 \mathrm{a}$ and $\mathrm{T} 4 \mathrm{~b}$, adding the concept of T4a $[6,7]$. T4a is defined as invading the serosa (in the anterior or posterior wall) or invading the visceral peritoneum (in the curvatures). Furthermore, the amount of subserosal fat tissue varies from person to person. Hence, the assessment of T4a varies depending on location and morphology [8-10]. It is difficult to predict T4a using only the morphological features of the tumor due to the above reasons. Therefore, making an accurate preoperative prediction of serosal invasion is quite challenging.

However, conventional computed tomography (CT) and endoscopic ultrasonography (EUS) are based on tumor morphology when evaluating the status of the serosa, which is inevitably limited by the above problems. Although EUS has certain advantages over CT in assessing $\mathrm{T}$ stage $[11,12]$, its results are obtained invasively and depend on operator experience without objective reflection on the overall staging (unable to accurately detect lymph nodes and distant metastases). Therefore, CT is still the most common staging tool for GC. In addition, studies on the quantitative analysis of GC using CT images have been widely carried out [13-17].

Recently, CT texture analysis that analyzes the distribution and relationship of pixel gray levels has developed rapidly [18]. Previous studies have confirmed that CT texture analysis and radiomics could predict $\mathrm{T}$ stages preoperatively in patients with advanced GC $[13,19,20]$. However, the postinjection delay of the arterial phase in these studies was 25-30 s. The major arteries can be clearly displayed in the early arterial phase (25-30 s), yet the mucosal layer may not be markedly enhanced simultaneously [21]. GC originates from the mucosal layer, so visualization of the mucosal lines is essential [22]. We assumed that the border of the gastric cancer would be displayed more clearly in the late arterial phase. In addition, various types of CT features, including morphology and CT values, can also be extracted [23, 24]. In clinical practice, a variety of clinicopathological information is collected prior to surgery, including endoscopic biopsy and multiple tumor markers. CT radiomics has been used to predict serosal invasion, but endoscopic biopsy and tumor markers were not included in the model [13]. Evaluating serosal invasion in GC is influenced by various factors. In recent years, GC staging has been predicted by integrating various types of preoperative clinicopathological information and radiographic findings $[25,26]$.

Thus, the purpose of our study was to develop and validate multivariate models integrating endoscopic biopsy, tumor markers, CT morphological characteristics, and $\mathrm{CT}$ value-related and texture parameters for predicting serosal invasion in GCs.

\section{Materials and methods}

This retrospective study used deidentified data without protected health information, and it was approved by the Ethical Committee of Nanjing Drum Tower Hospital (Approval Documents Number: 2020-032-01). The requirement for informed consent was waived.

\section{Patients}

Between April 2019 and April 2020, we searched the radiologic image archives of our hospital consecutively to identify 231 patients who had GC diagnosed by histopathologic analysis. The inclusion criteria were as follows: (1) pathological confirmation of GC postoperatively and (2) the availability of endoscopic biopsy, tumor markers, and abdominal contrast-enhanced CT within 2 weeks prior to surgery [25]. The exclusion criteria were as follows: (1) a history of GC treatment preoperatively $(n=8)$; (2) lacking $40 \mathrm{~s}$ LAP information $(n=24)$; (3) insufficient distention of the stomach $(n=18)$; (4) poor imaging quality due to respiratory or peristaltic motion $(n=7)$; (5) hardly visible due to the small size of the GC on CT images (long diameter $<1 \mathrm{~cm}) \quad(n=17)$; and (6) incomplete information on tumor markers $(n=3)$.

Ultimately, 154 patients (male, 107; female, 478; median age, 64 years; age range, 30-78 years) were included. The patients were divided into primary and validation cohorts based on the time of surgery at a ratio of 2:1. The flow chart of patient selection is shown in Fig. 1. The overall framework of this study is shown in Fig. 2. In addition, we added an extra cohort consisting of advanced GCs with negative tumor markers (83 patients).

\section{Endoscopic biopsy}

Information on histological differentiation based on preoperative endoscopic biopsy was retrospectively examined and recorded by a pathologist (with 7 years of experience in pathological diagnosis of the digestive system) according to the WHO Classification of Tumors of the Digestive System (2019 version) [25, 27]. The tumors 


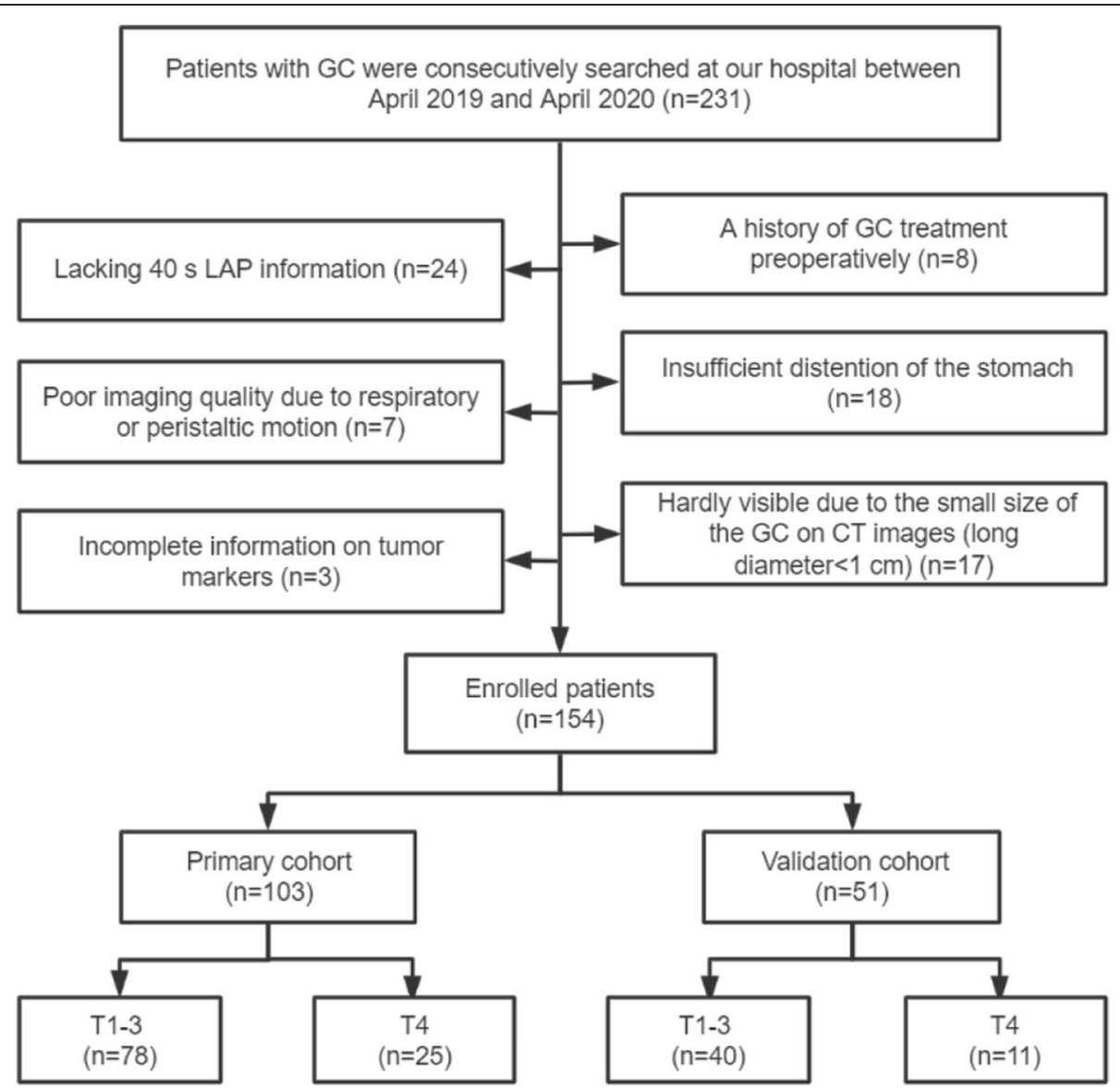

Fig. 1 The flowchart of the patients enrolled in our study. GC, gastric cancer; LAP, late arterial phase; CT, computed tomography

were classified into two groups: group 1, poor differentiation; group 2, moderate/well differentiation.

\section{Tumor markers}

Six serum tumor markers, including alpha fetoprotein (AFP), carcinoembryonic antigen (CEA), carbohydrate antigen (CA) 125, CA199, CA724, and CA242, were collected within 2 weeks before surgery [25].

\section{CT image acquisition}

CT examinations were performed on a 64-row scanner (uCT 780, United Imaging, Shanghai, China). All patients were required to fast for at least $6 \mathrm{~h}$ and take 600$1000 \mathrm{~mL}$ of warm water orally prior to the examination. All patients were placed in the supine position, and the scan covered the upper or entire abdomen. Following the nonenhanced scan, $1.5 \mathrm{~mL} / \mathrm{kg}$ iodinated contrast agent (Omnipaque $350 \mathrm{mg} \mathrm{I} / \mathrm{mL}$, GE Healthcare) was injected intravenously at a flow rate of $3.0 \mathrm{~mL} / \mathrm{s}$ using a high-pressure syringe. Imaging was achieved with postinjection delays of 40,70, and $180 \mathrm{~s}$ after the initiation of contrast material injection, corresponding to the late arterial, portal, and delayed phases, respectively. The CT scan parameters were as follows: tube voltage $120 \mathrm{kV}$, tube current $150-250 \mathrm{~mA}$, field of view $35-50 \mathrm{~cm}$, matrix $512 \times 512$, rotation time $0.7 \mathrm{~s}$, and pitch 1.0875 . The images were reconstructed with section thicknesses of 1 and $5 \mathrm{~mm}$; the former were used for multiplanar reconstruction, and the latter were used for the measurement of CT values due to the signal-to-noise ratio [25].

\section{Image analysis Morphological characteristics}

Readers 1 and 2 (with 5 and 7 years of experience in abdominal imaging, respectively), who were blinded to the clinicopathological information of the patients except for the general location of the tumors, independently evaluated the morphological characteristics of each lesion on the $40 \mathrm{~s}$ LAP CT images, and their results were used to assess interobserver agreement. Any discrepant opinions between readers 1 and 2 were resolved by reader 3 (with 20 years of experience in abdominal imaging) as the final result [25]. The characteristics were: 1) major location (cardia, body, and antrum); 2) tumor range (1 location, $\geq 2$ locations); 3) major orientation (lesser curvature, greater curvature, anterior wall, and posterior wall); 4) 


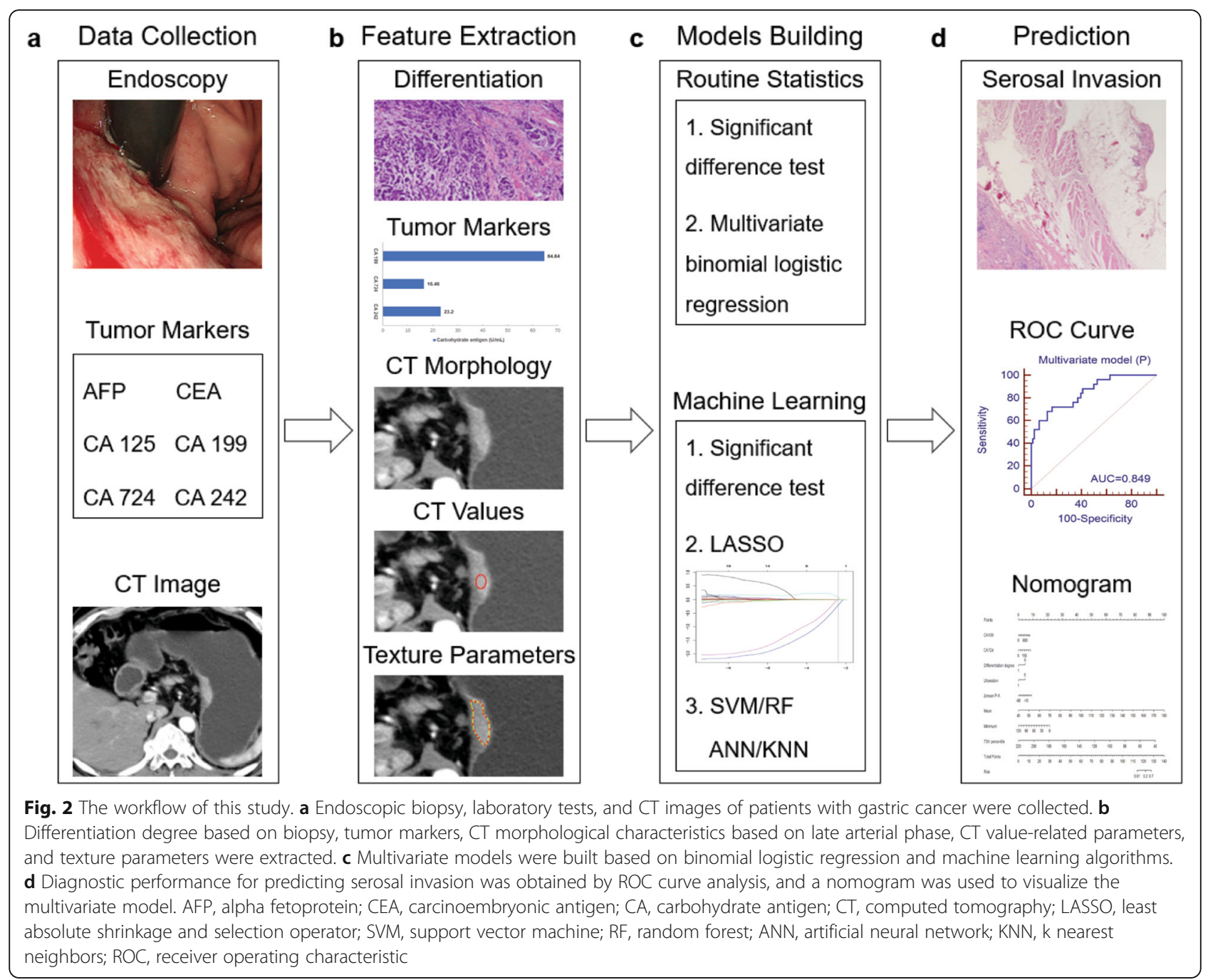

circumferential range $(1 / 4,2 / 4,3 / 4$, and $4 / 4) ; 5)$ infiltrative growth (absent, present): unclear border between the lesion and the normal gastric wall; 6) ulceration (absent, present); 7) adjacent adipose tissue stains (absent, present); 8) mucosal line status (interruption, thickening); 9) morphological type (thickening type, mass type); 10) linitis plastica (absent, present); and 11) lymphadenectasis (absent, present): the short axis of the regional lymph node was greater than $1 \mathrm{~cm}$.

\section{$C T$ value-related parameters}

The oval regions of interest (ROIs) were drawn to encompass the area of greatest enhancement on the maximal section in $40 \mathrm{~s}$ LAP and were copied on the same slice in the other three phases by reader 1 . The mean size of the ROIs was $32.82 \mathrm{~mm}^{2}$, and the range was $6.60-156.90 \mathrm{~mm}^{2}$. The mean CT attenuation values of the tumor in the nonenhanced, late arterial, portal, and delayed phases were recorded as the $\mathrm{N}$ value mean, AP value mean, $\mathrm{PP}$ value mean, and $\mathrm{DP}$ value mean, respectively, as well as the maximum and minimum $\mathrm{CT}$ values. With the N, AP, and PP value means as the references, postcontrast tumorous attenuation differences $(\Delta$ mean A-N, $\Delta$ mean P-N, $\Delta$ mean D-N, $\Delta$ mean P-A, $\Delta$ mean $\mathrm{D}-\mathrm{A}$, and $\Delta$ mean $\mathrm{D}-\mathrm{P})$ were calculated. CT value-related parameters derived from the ROIs delineated by reader 1 were used to predict serosal invasion. To determine the interobserver reproducibility, reader 2 repeated the above procedure [25].

\section{CT texture analysis}

The LAP CT images were uploaded into in-house software (Image Analyzer 2.0, China). All the images were reviewed by reader 1 . Polygonal ROIs (mean size, 402.23 $\mathrm{mm}^{2}$; range, $24.36-2442.87 \mathrm{~mm}^{2}$ ) were manually drawn along the margin of the tumor on the largest crosssection (Fig. 3), avoiding the normal gastric wall tissue and the gastric cavity contents. Texture parameters were as follows: (1) the first-order features included the mean, standard deviation, max frequency, mode, minimum, 


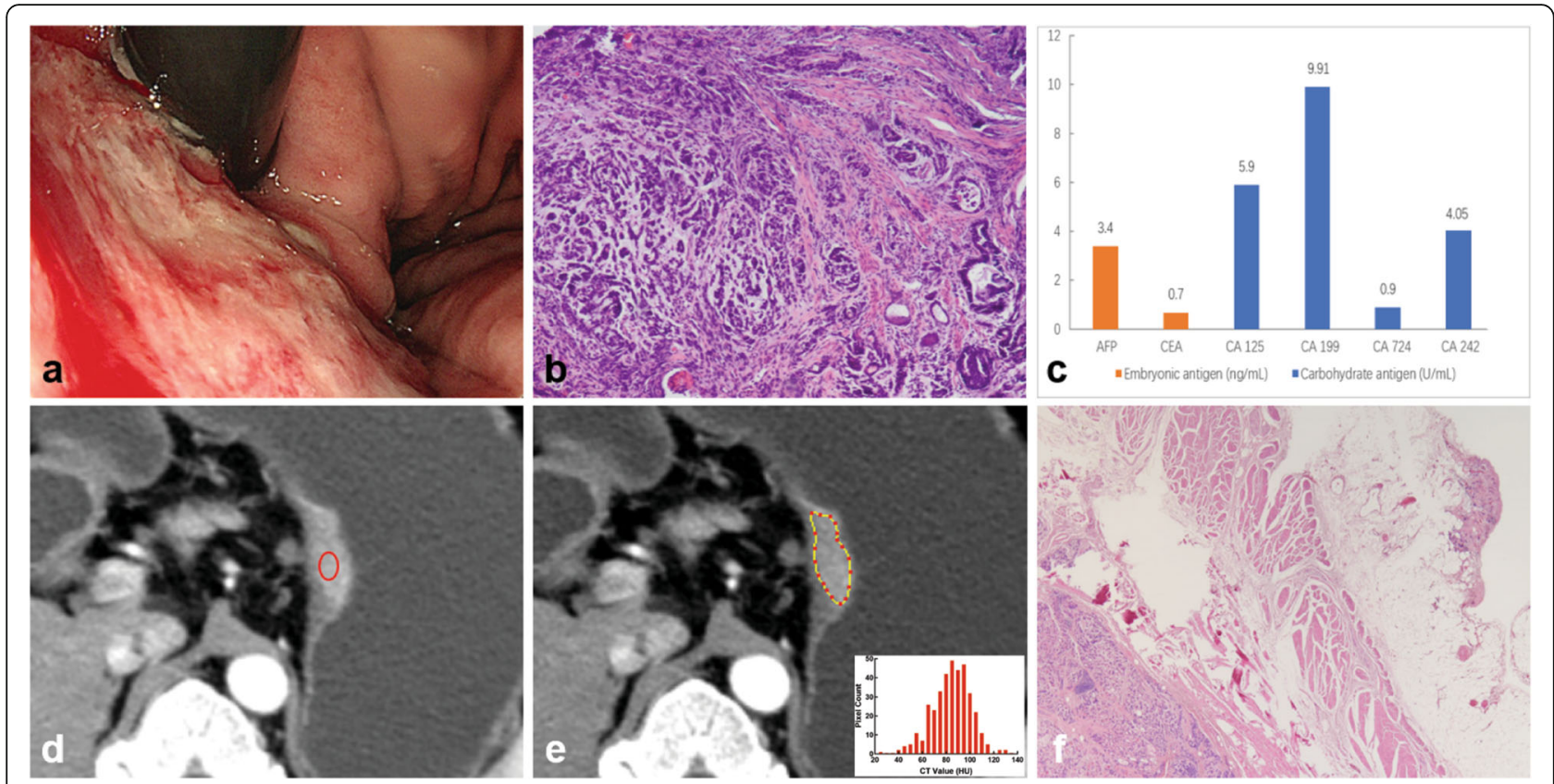

Fig. 3 A 50-year-old man with gastric cancer pathologically diagnosed with serosal invasion. a The endoscopic image indicates a mass lesion in the posterior wall of the stomach body. $\mathbf{b}$ Hematoxylin and eosin (H\&E) staining of a specimen based on endoscopic biopsy shows a poorly differentiated carcinoma (original magnification, $\times 100$ ). c The values of the tumor markers, including AFP, CEA, CA125, CA199, CA724, and CA242, were $3.40 \mathrm{ng} / \mathrm{mL}, 0.70 \mathrm{ng} / \mathrm{mL}, 5.90 \mathrm{U} / \mathrm{mL}, 9.91 \mathrm{U} / \mathrm{mL}, 0.90 \mathrm{U} / \mathrm{mL}$, and $4.05 \mathrm{U} / \mathrm{mL}$, respectively. $\mathbf{d}$ The late arterial phase computed tomography (CT) image shows a mass lesion with marked enhancement in the posterior wall of the stomach body. An oval region of interest (ROI) was drawn to encompass the area of greatest enhancement on the maximal section, and the CT value-related parameters were extracted. e A polygonal ROI was manually drawn along the margin of the tumor on the largest cross-section, and the texture parameters were extracted. $\mathbf{f} \mathrm{H} \& \mathrm{E}$ staining of a postoperative specimen confirms gastric cancer with serosal invasion (original magnification, $\times 20$ ). AFP, alpha fetoprotein; CEA, carcinoembryonic antigen; CA, carbohydrate antigen

maximum, cumulative percentiles (the 5th, 10th, 25th, 50th, 75th, and 90th percentiles), skewness, kurtosis, entropy, and histogram width; (2) the second-order features were from the gray-level cooccurrence matrix (GLCM) and included Entropy GLCM, Energy GLCM, Inertia GLCM, and Variance GLCM. Texture parameters derived from the ROIs delineated by reader 1 were used to predict serosal invasion. To determine the interobserver reproducibility, reader 2 repeated the above procedure [25].

\section{Development, performance, and validation of the multivariate models}

Significant $(P<0.05)$ variables in the univariate analysis were input into a multivariate binomial logistic regression based on a backward elimination process in the primary cohort. The Hosmer-Lemeshow test was used to measure the goodness of fit. A nomogram was constructed based on the multivariate model in the primary cohort with the R software package (version 3.5.2: http:// www.Rproject.org). The multivariate model was applied to the validation cohort and the extra cohort. The diagnostic efficacy was evaluated with receiver operating characteristic (ROC) curve analysis [25].
If significance $(P<0.05)$ was met in the univariate analysis of the primary cohort, the variables were incorporated into the least absolute shrinkage and selection operator (LASSO) for dimension reduction. Then, we built four machine learning models, including support vector machine (SVM), random forest (RF), artificial neural network (ANN) and $k$ nearest neighbors (KNN), with the LASSO selected features as the input factors. The fivefold crossvalidation was performed to improve and compare these models' performances. The best performing model was selected to build the predictive model for serosal invasion and applied to the validation cohort [25, 28].

\section{Pathological assessment after surgery}

All patients underwent gastrectomy (either total or partial). All gastric specimens were processed according to standard pathological procedures. The pathological $\mathrm{T}$ stage was retrospectively examined and recorded according to the 8th American Joint Committee on Cancer classification $[7,25]$. The patients were divided into two groups (T1-3 vs. T4). 


\section{Statistical analysis}

The differences in demographic data, endoscopic biopsy, and morphological characteristics were assessed with the chi-square or Fisher's exact test $(n<5)$. Kappa statistics were applied to evaluate the interobserver consistency. A kappa value of less than 0.200 was considered poor, 0.201-0.400 was considered fair, $0.401-0.600$ was considered moderate, $0.601-0.800$ was considered good, and 0.801-1.000 was considered excellent. The normality distributions of the tumor markers, CT value-related parameters, and texture parameters were evaluated by the Shapiro-Wilk test. Based on the normality test results, the differences between T1-3 and T4 were analyzed by the Mann-Whitney $U$ test. ROC curve analysis was performed, and the area under the ROC curve (AUC), diagnostic sensitivity, specificity, and accuracy were calculated. The cutoff value was established by calculating the largest Youden index (Youden index = sensitivity+ specificity-1). The interobserver agreement of the CT value-related and texture parameters was estimated with the intraclass correlation coefficient (ICC) (0.000-0.200: poor; 0.201-0.400: fair; 0.401-0.600: moderate; 0.6010.800: good; 0.801-1.000: excellent). All statistical analyses were performed with SPSS (version 22.0 for Microsoft Windows $\times 64$, SPSS), MedCalc Statistical Software (version 11.4.2.0 MedCalc Software bvba; http://www.medcalc.org; 2011), and R software (version 3.5.2: http://www.Rproject.org). A two-tailed $P$ value $<0.05$ was considered statistically significant [25].

\section{Results}

\section{Qualitative analysis}

Table 1 summarizes the results of the univariate analysis of the demographic data, endoscopic biopsy, and morphological characteristics between the $\mathrm{T} 1-3$ and $\mathrm{T} 4$ groups in the primary and validation cohorts. The differentiation degree, infiltrative growth, ulceration, and morphological type differed significantly between the two groups in the primary cohort (all $P<0.05$ ). There were no significant differences in major location, tumor range, major orientation, circumferential range, adjacent adipose tissue stains, mucosal line status, linitis plastica, or lymphadenectasis between the two groups in the primary cohort (all $P>0.05)$. There were significant differences for the six characteristics in the validation cohort (all $P<0.05$ ).

\section{Quantitative analysis}

\section{Tumor markers}

The values of CA199, CA724, and CA242 differed significantly in the primary cohort $(P=0.007,0.023$, and 0.015 , respectively, Table 2 ). There were no significant differences in CEA, CA125, or AFP between the T1-3 and T4 groups in the primary cohort (all $P>0.05$, Table A1).

\section{$C T$ value-related and texture parameters}

The results of the univariate analysis for quantitative CT value-related and texture parameters between the T1-3 and T4 groups in the primary cohort are shown in Table 2. For the CT value-related parameters, there were significant differences in $\mathrm{AP}$ value mean, $\mathrm{AP}$ value min, $\Delta$ mean $\mathrm{A}-\mathrm{N}$, $\Delta$ mean $\mathrm{P}-\mathrm{A}$, and $\Delta$ mean $\mathrm{D}-\mathrm{A}($ all $P<0.05)$. For the texture parameters, nine parameters differed significantly between the two groups (all $P<0.05$ ), and the corresponding AUCs ranged from 0.633 to 0.742 (Table 3 ).

\section{Development, performance, and validation of the multivariate models Multivariate binomial logistic regression}

The best-performing model based on regression for predicting serosal invasion in the primary cohort consisted of differentiation degree, ulceration, CA199, CA724, $\Delta$ mean P-A, mean, minimum, and 75 th percentile. The multivariate model had a predictive ability with a cutoff of 0.24 ( $\mathrm{AUC}=0.849, P<0.001$ ), which yield a sensitivity, specificity, and accuracy of $72.0,83.3$, and $80.6 \%$, respectively. The ROC curve of the primary cohort is plotted in Fig. 4. The cutoff value of 0.24 was used to test the predictive performance of the validation cohort, which yield a sensitivity, specificity, and accuracy of 81.8 , 82.5 , and $82.4 \%$, respectively. A nomogram constructed based on the multivariate logistic regression model in the primary cohort for predicting serosal invasion is displayed in Fig. 5.

In addition, the cutoff value of 0.24 was used to test the predictive performance of the extra cohort consisting of advanced GCs with negative tumor markers (83 patients), which yield a sensitivity, specificity, and accuracy of $53.3,76.5$, and $72.3 \%$, respectively.

\section{Machine learning algorithms}

LASSO was applied to reduce the dimensions and to select optimal variables in the primary cohort (Fig. 6). Finally, infiltrative growth, ulceration, CA242, CA724, and minimum were integrated to build multivariate models using the SVM, RF, ANN, and KNN algorithms. The multivariate model generated by RF showed best performance in the four machine learning algorithms with an AUC of 0.865. The developed model based on RF was also applied in the validation cohort and achieved an AUC of 0.845 .

\section{Interobserver agreement}

All CT morphological characteristics showed good to excellent interobserver agreement in the evaluation of GCs $(\kappa=0.715-0.902)$ (Table A2). All CT value-related parameters $(\mathrm{ICC}=0.687-0.941)$ and texture parameters (ICC $=0.706-0.989)$ also showed good to excellent interobserver agreement (Tables A3 and A4). 
Table 1 Univariate analysis of demographic data, endoscopic biopsy, and morphological characteristics in the primary and validation cohorts

\begin{tabular}{|c|c|c|c|c|c|c|}
\hline \multirow[t]{2}{*}{ Characteristics } & \multicolumn{2}{|c|}{ Primary cohort } & \multirow[t]{2}{*}{$P$ value } & \multicolumn{2}{|c|}{ Validation cohort } & \multirow[t]{2}{*}{$P$ value } \\
\hline & T1-3 $(n=78)$ & $\mathrm{T} 4(n=25)$ & & $\mathrm{T} 1-3(n=40)$ & $\mathrm{T} 4(n=11)$ & \\
\hline \multicolumn{7}{|l|}{ Demographic data } \\
\hline Gender & & & 0.154 & & & 0.214 \\
\hline Male & 58 & 22 & & 23 & 4 & \\
\hline Female & 20 & 3 & & 17 & 7 & \\
\hline Age (y) & & & 0.543 & & & 1.000 \\
\hline$<60$ & 29 & 11 & & 13 & 3 & \\
\hline$\geq 60$ & 49 & 14 & & 27 & 8 & \\
\hline \multicolumn{7}{|l|}{ Endoscopic biopsy } \\
\hline Differentiation degree & & & $0.004^{*}$ & & & 0.141 \\
\hline Poor & 48 & 23 & & 26 & 10 & \\
\hline Moderate \& Well & 30 & 2 & & 14 & 1 & \\
\hline \multicolumn{7}{|l|}{ Morphological characteristics } \\
\hline Major location & & & 0.884 & & & 0.690 \\
\hline Cardia & 21 & 6 & & 14 & 2 & \\
\hline Body & 27 & 8 & & 14 & 5 & \\
\hline Antrum & 30 & 11 & & 12 & 4 & \\
\hline Tumor range & & & 0.121 & & & $0.009^{*}$ \\
\hline 1 location & 51 & 12 & & 32 & 4 & \\
\hline$\geq 2$ locations & 27 & 13 & & 8 & 7 & \\
\hline Major orientation & & & 0.637 & & & 0.591 \\
\hline Lesser curvature & 35 & 11 & & 19 & 7 & \\
\hline Greater curvature & 2 & 2 & & 3 & 0 & \\
\hline Anterior wall & 7 & 2 & & 2 & 1 & \\
\hline Posterior wall & 34 & 10 & & 16 & 3 & \\
\hline Circumferential range & & & 0.428 & & & $0.010^{*}$ \\
\hline $1 / 4$ & 34 & 7 & & 24 & 1 & \\
\hline $2 / 4$ & 26 & 11 & & 10 & 7 & \\
\hline $3 / 4$ & 11 & 3 & & 3 & 1 & \\
\hline $4 / 4$ & 7 & 4 & & 3 & 2 & \\
\hline Infiltrative growth & & & $0.009^{*}$ & & & $0.001^{*}$ \\
\hline Absent & 54 & 10 & & 30 & 2 & \\
\hline Present & 24 & 15 & & 10 & 9 & \\
\hline Ulceration & & & $0.036^{*}$ & & & 0.305 \\
\hline Absent & 20 & 12 & & 20 & 8 & \\
\hline Present & 58 & 13 & & 20 & 3 & \\
\hline Adjacent adipose tissue stains & & & 0.267 & & & $0.009^{*}$ \\
\hline Absent & 56 & 15 & & 25 & 2 & \\
\hline Present & 22 & 10 & & 15 & 9 & \\
\hline Mucosal line status & & & 0.280 & & & $0.002^{*}$ \\
\hline Interruption & 28 & 12 & & 11 & 9 & \\
\hline Thickening & 50 & 13 & & 29 & 2 & \\
\hline
\end{tabular}


Table 1 Univariate analysis of demographic data, endoscopic biopsy, and morphological characteristics in the primary and validation cohorts (Continued)

\begin{tabular}{|c|c|c|c|c|c|c|}
\hline \multirow[t]{2}{*}{ Characteristics } & \multicolumn{2}{|c|}{ Primary cohort } & \multirow[t]{2}{*}{$P$ value } & \multicolumn{2}{|c|}{ Validation cohort } & \multirow[t]{2}{*}{$P$ value } \\
\hline & $\mathrm{T} 1-3(n=78)$ & $\mathrm{T} 4(n=25)$ & & $\mathrm{T} 1-3(n=40)$ & T4 $(n=11)$ & \\
\hline Morphological type & & & $0.026^{*}$ & & & $0.037^{*}$ \\
\hline Thickening type & 12 & 9 & & 11 & 7 & \\
\hline Mass type & 66 & 16 & & 29 & 4 & \\
\hline Linitis plastica & & & 0.091 & & & 0.292 \\
\hline Absent & 76 & 22 & & 37 & 9 & \\
\hline Present & 2 & 3 & & 3 & 2 & \\
\hline Lymphadenectasis & & & 1.000 & & & 0.598 \\
\hline Absent & 66 & 21 & & 36 & 9 & \\
\hline Present & 12 & 4 & & 4 & 2 & \\
\hline
\end{tabular}

\section{Discussion}

In the current study, we investigated the ability of multivariate models integrating preoperative clinicopathological features and radiographic findings based on LAP CT images to predict serosal invasion in GC. To build the multivariate models, the differentiation degree based on endoscopic biopsy, 6 tumor markers, 11 CT morphological characteristics, $18 \mathrm{CT}$ value-related parameters, and $32 \mathrm{CT}$ texture parameters were collected. There were significant differences in multiple features between the T1-3 and T4 groups.

Endoscopic biopsy and tumor markers are widely used in the early diagnosis and disease monitoring in gastric cancer $[5,29,30]$. In this study, we demonstrated that

Table 2 Statistical description and univariate analysis of tumor markers, the CT value-related parameters, and texture parameters in the primary cohort

\begin{tabular}{|c|c|c|c|}
\hline Parameters & T1-3 & T4 & $P$ value \\
\hline \multicolumn{4}{|l|}{ Tumor markers } \\
\hline CA199 (U/mL) & $8.92(5.55,12.91)$ & $11.74(8.39,197.35)$ & $0.007^{*}$ \\
\hline CA724 (U/mL) & $1.83(1.20,4.69)$ & $3.00(1.73,22.22)$ & $0.023^{*}$ \\
\hline CA242 (U/mL) & $3.19(2.21,5.54)$ & $6.91(2.69,105.51)$ & $0.015^{*}$ \\
\hline \multicolumn{4}{|c|}{$\mathrm{CT}$ value-related parameters } \\
\hline AP value mean $(\mathrm{HU})$ & $112.51(89.22,137.63)$ & $94.12(76.98,113.02)$ & $0.024^{*}$ \\
\hline AP value $\min (H U)$ & $90.00(71.50,113.00)$ & $70.00(54.50,101.50)$ & $0.018^{*}$ \\
\hline$\Delta$ mean A-N (HU) & $69.86(50.52,96.24)$ & $50.28(34.13,75.73)$ & $0.028^{*}$ \\
\hline$\Delta$ mean P-A (HU) & $-19.02(-35.97,-6.23)$ & $-6.12(-24.34,7.84)$ & $0.024^{*}$ \\
\hline$\Delta$ mean D-A (HU) & $-32.65(-50.80,-10.62)$ & $-17.50(-36.10,14.62)$ & $0.021^{*}$ \\
\hline \multicolumn{4}{|l|}{ Texture parameters } \\
\hline Mean $(H U)$ & $106.47(92.20,120.32)$ & $90.74(71.18,111.48)$ & $0.027^{*}$ \\
\hline Max frequency & $15.50(10.00,29.00)$ & $31.00(19.00,51.00)$ & $<0.001^{*}$ \\
\hline Minimum $(\mathrm{HU})$ & $58.00(41.75,75.25)$ & $42.00(25.50,55.00)$ & $0.006^{*}$ \\
\hline 5th percentile $(\mathrm{HU})$ & $76.50(63.50,93.00)$ & $63.00(45.00,79.50)$ & $0.015^{*}$ \\
\hline 10th percentile (HU) & $82.00(68.00,101.00)$ & $68.00(51.00,89.00)$ & $0.019^{*}$ \\
\hline 25th percentile $(\mathrm{HU})$ & $93.00(79.75,111.00)$ & $78.00(59.50,98.50)$ & $0.017^{*}$ \\
\hline 50th percentile $(\mathrm{HU})$ & $106.00(91.75,120.50)$ & $90.00(68.50,109.50)$ & $0.029^{*}$ \\
\hline 75th percentile (HU) & $118.50(103.00,133.25)$ & $101.00(84.50,123.50)$ & $0.027^{*}$ \\
\hline 90th percentile (HU) & $129.00(114.75,147.00)$ & $114.00(95.50,141.00)$ & $0.046^{*}$ \\
\hline
\end{tabular}

The data are presented as median with (1st quartile, 3rd quartile)

$C A$ carbohydrate antigen, $A P$ arterial phase

${ }^{*} P<0.05$ with Mann-Whitney $U$ test 
Table 3 The diagnostic performance of tumor markers, the $C T$ value-related parameters, and texture parameters in the primary cohort

\begin{tabular}{|c|c|c|c|c|c|c|}
\hline Parameters & Cutoff & Sensitivity & Specificity & AUC & Accuracy & $P$ value \\
\hline \multicolumn{7}{|l|}{ Tumor markers } \\
\hline CA199 (U/mL) & 9.47 & 0.720 & 0.615 & 0.681 & 0.640 & $0.009^{*}$ \\
\hline CA724 (U/mL) & 1.48 & 0.840 & 0.436 & 0.652 & 0.534 & $0.022^{*}$ \\
\hline CA242 (U/mL) & 6.30 & 0.520 & 0.821 & 0.662 & 0.748 & $0.021^{*}$ \\
\hline \multicolumn{7}{|c|}{ CT value-related parameters } \\
\hline AP value mean (HU) & 96.62 & 0.680 & 0.692 & 0.651 & 0.689 & $0.031^{*}$ \\
\hline AP value min $(H U)$ & 78.00 & 0.680 & 0.680 & 0.658 & 0.680 & $0.024^{*}$ \\
\hline$\Delta$ mean $\mathrm{A}-\mathrm{N}(\mathrm{HU})$ & 54.11 & 0.640 & 0.705 & 0.647 & 0.689 & $0.035^{*}$ \\
\hline$\Delta$ mean $\mathrm{P}-\mathrm{A}(\mathrm{HU})$ & -7.46 & 0.560 & 0.744 & 0.650 & 0.699 & $0.025^{*}$ \\
\hline$\Delta$ mean D-A (HU) & -25.09 & 0.680 & 0.603 & 0.654 & 0.622 & $0.021^{*}$ \\
\hline \multicolumn{7}{|l|}{ Texture parameters } \\
\hline Mean $(H U)$ & 90.74 & 0.520 & 0.782 & 0.648 & 0.718 & $0.036^{*}$ \\
\hline Max frequency & 20.00 & 0.760 & 0.680 & 0.742 & 0.699 & $<0.001^{*}$ \\
\hline Minimum (HU) & 56.00 & 0.800 & 0.539 & 0.682 & 0.602 & $0.005^{*}$ \\
\hline 5th percentile $(\mathrm{HU})$ & 69.00 & 0.640 & 0.680 & 0.662 & 0.670 & $0.014^{*}$ \\
\hline 10th percentile (HU) & 70.00 & 0.560 & 0.744 & 0.657 & 0.699 & $0.021^{*}$ \\
\hline 25th percentile (HU) & 89.00 & 0.720 & 0.603 & 0.659 & 0.631 & $0.021^{*}$ \\
\hline 50th percentile (HU) & 90.00 & 0.520 & 0.769 & 0.645 & 0.709 & $0.038^{*}$ \\
\hline 75th percentile (HU) & 101.00 & 0.520 & 0.782 & 0.647 & 0.718 & $0.038^{*}$ \\
\hline 90th percentile (HU) & 107.00 & 0.440 & 0.846 & 0.633 & 0.747 & 0.064 \\
\hline
\end{tabular}

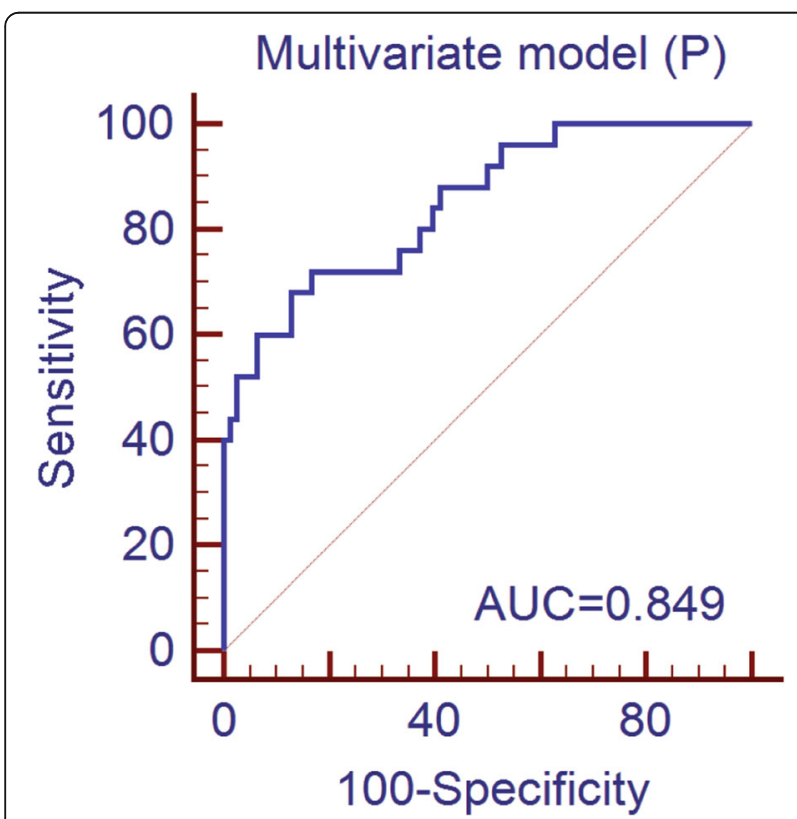

Fig. 4 The receiver operating characteristic (ROC) curve of the multivariate model based on binomial logistic regression analysis for predicting the serosal invasion of gastric cancer in the primary cohort. The AUC of the multivariate model was 0.849. AUC, area under the receiver operating characteristic curve the differentiation degree based on biopsy, CA199, CA724, and CA242 were able to predict serosal invasion in GC. Poorly differentiated GC is usually more aggressive and carries a higher risk of deeper invasion. Kim DK et al. reported that the deep submucosal invasion of GC was related to the poorly differentiated type [31]. This was consistent with our results. Additionally, the tumor markers indirectly reflect the changes in related gene expression during tumor progression. A previous study reported that preoperative serum CA 242 values can serve as an independent prognostic marker for GC patients [30].

We found that the morphological characteristics in 40s LAP, including infiltrative growth, ulceration, and morphological type, differed significantly between the T1-3 and T4 groups in the primary cohort. When accompanied by infiltrative growth, ulceration, and thickening type, GC is more aggressive and more likely to invade the serosa. However, there were significant differences in six characteristics in the validation cohort. The different results of the morphological characteristics analysis in the primary and validation cohorts could be explained by the different sample sizes. The primary cohort had a larger sample size and thus might be more representative. 


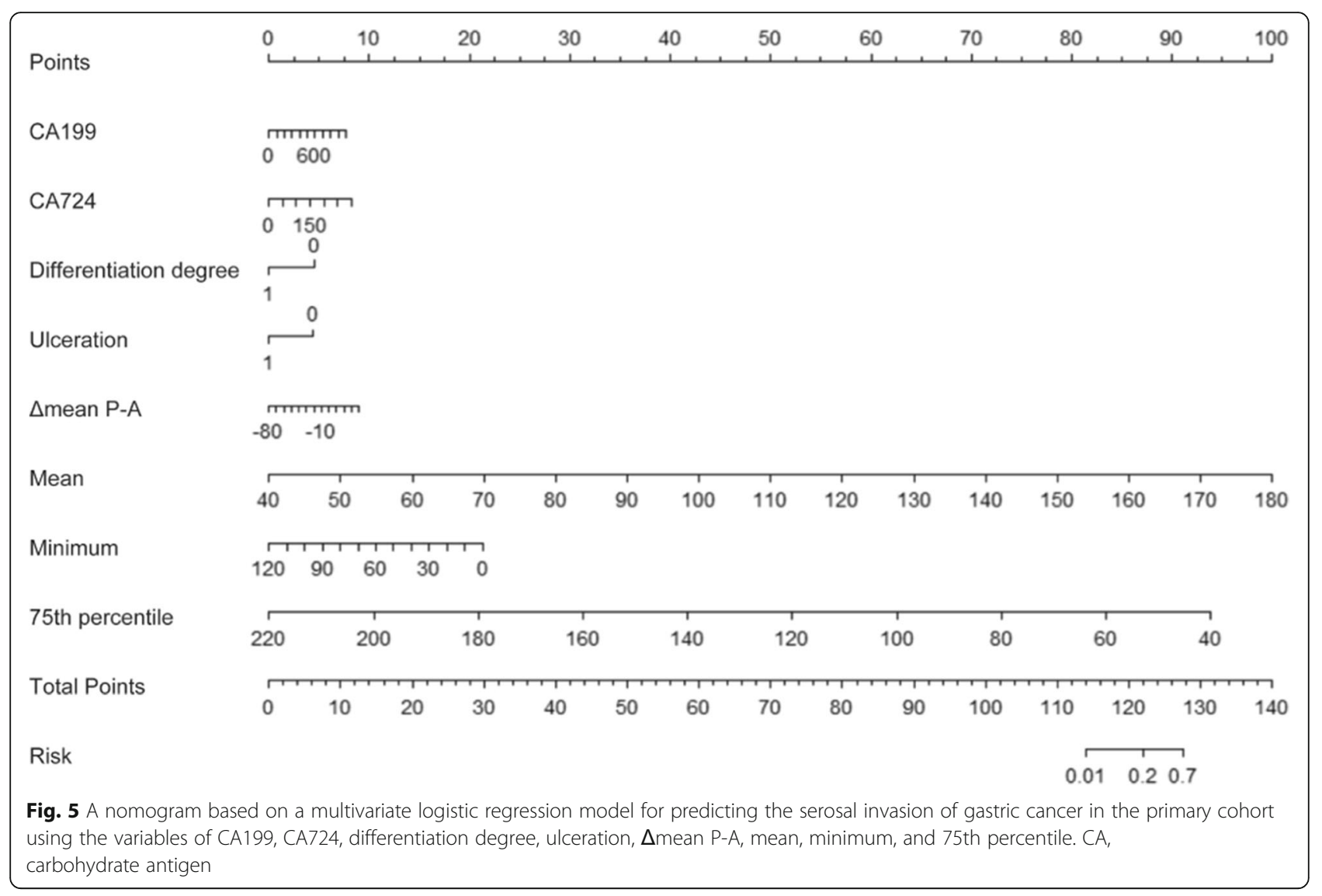

In quantitative analysis, we used not only $\mathrm{CT}$ valuerelated parameters but also texture parameters. For the CT value-related parameters, there were significant differences in AP value mean, AP value min, $\Delta$ mean $\mathrm{A}-\mathrm{N}$, $\Delta$ mean $\mathrm{P}-\mathrm{A}$, and $\Delta$ mean $\mathrm{D}-\mathrm{A}$ between the $\mathrm{T} 1-3$ and $\mathrm{T} 4$ groups in the primary cohort. This indicates that the parameters related to the LAP may better reflect tumor information. Furthermore, nine texture parameters, including the mean, max frequency, minimum, and 5th-90th percentiles, differed significantly in the primary cohort. GCs with serosal invasion had a higher max frequency value (indicating the peak value of the histogram). The 5th-90th percentiles reflected the enhancement degree of different components of the tumor. GCs with serosal invasion tend to be more aggressive and grow rapidly, resulting in insufficient blood supply and necrosis [32].

To develop multivariate models for predicting serosal invasion in $\mathrm{GC}$, the regression analysis and machine learning algorithms were used in the study. For the regression model, we utilized a backward elimination process [33]. For the machine learning algorithm, LASSO was used for dimension reduction, which is in general use $[13,17,34]$. The multivariate models based on regression analysis and the RF algorithm showed satisfactory performance in the primary cohort $(\mathrm{AUC}=0.849$ and 0.865 , respectively). The AUC of multivariate model generated by RF was slightly better than regression analysis. Furthermore, we applied the Delong test to compare the performance of the models based on RF and regression analysis and found that there was no significant difference between the two models. The developed models were also used in the validation cohort and achieved better performance.

In addition, the cutoff value of 0.24 (the same as the regression model developed in the primary cohort) was used to test the predictive performance of the extra cohort consisting of advanced GCs with negative tumor markers (83 patients), which yield an accuracy of $72.3 \%$.

Most previous works applied to predict serosal invasion in GC focused on morphology [8-10, 35]. Although the number of studies was abundant, the results varied widely and were controversial. In recent years, CT radiomics has been used to predict serosal invasion, while endoscopic biopsy and tumor markers were not included in the model building [13]. Evaluating serosal invasion in $\mathrm{GC}$ is influenced by various factors. In this situation, to solve the above problems, a comprehensive evaluation based on preoperative clinicopathological features and 

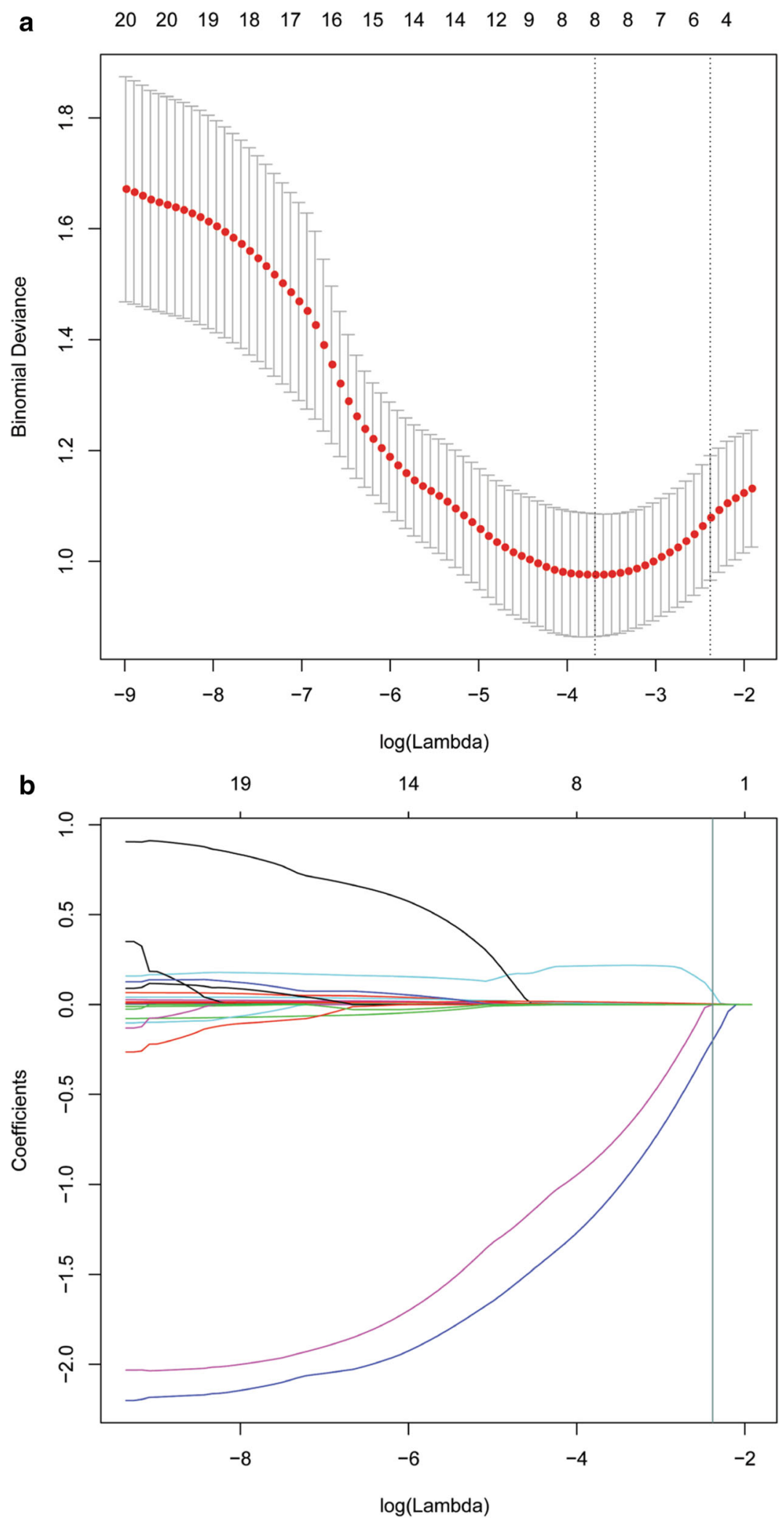

Fig. 6 (See legend on next page.) 
(See figure on previous page.)

Fig. 6 Feature selection was performed using the least absolute shrinkage and selection operator (LASSO) regression model. a Tuning parameter $(\lambda)$ selection in the LASSO model used fivefold cross-validation via minimum criteria. Vertical lines were drawn at the optimal values using the minimum criteria and 1 standard error of the minimum criteria. The optimal $\lambda$ value of 0.0922 with $\log (\lambda)=-2.3838$ was chosen. $\mathbf{b}$ LASSO coefficient profiles of the 21 selected features. A coefficient profile plot was generated versus the selected log $(\lambda)$ value using fivefold crossvalidation; five selected features with nonzero coefficients were retained

radiographic findings was adopted, and it achieved a satisfactory result. It is worth mentioning that the interobserver agreement for all morphological characteristics, CT value-related parameters, and texture features proved to be good or excellent.

There are some limitations of this study. First, it used retrospective data collected from a single center, and the sample might be biased. Second, the texture features were derived from the two-dimensional ROIs of lesions with manual segmentation, which might have lost feature information in the longitudinal direction. Third, only the biopsy information was extracted, and more detailed information needs to be included from endoscopy. Thus, additional investigation to remedy the abovementioned insufficiencies should be performed.

In conclusion, we developed and validated multivariate models integrating preoperative clinicopathological features and radiographic findings based on LAP CT images to predict serosal invasion in GCs, and it achieved a favorable performance.

\section{Abbreviations \\ AFP: Alpha fetoprotein; ANN: Artificial neural network; AP: Arterial phase; AUC: Area under the curve; CA: Carbohydrate antigen; \\ CEA: Carcinoembryonic antigen; CT: Computed tomography; DP: Delayed phase; EUS: Endoscopic ultrasonography; GC: Gastric cancer; GLCM: Gray- level cooccurrence matrix; ICC: Intraclass correlation coefficient; KNN: K nearest neighbors; LAP: Late arterial phase; LASSO: Least absolute shrinkage and selection operator; N: Non-enhanced; PP: Portal phase; RF: Random forest; ROC: Receiver operating characteristic; ROls: Regions of interest; SVM: Support vector machine}

\section{Supplementary Information}

The online version contains supplementary material available at https:/doi. org/10.1186/s12885-021-08672-0.

Additional file 1: Table A1. Statistical description and univariate analysis of tumor markers, the $C \mathrm{~T}$ value-related parameters, and texture parameters in the primary cohort $(P>0.05)$. Table A2. Interobserver agreement for CT morphological characteristics. Table A3. Interobserver agreement for $\mathrm{CT}$ value-related parameters. Table A4. Interobserver agreement for texture parameters.

Acknowledgements

Not applicable.

\section{Authors' contributions}

Conceptualization: S.L. and Z.Y.Z.; Data curation: X.M.Q. and C.F.J.; Formal analysis: X.M.Q., C.F.J. and M.Y.X.; Funding acquisition: S.L.; Methodology and Project administration: S.L. and L.L.; Resources and Supervision: L.L., S.L., and Z.Y.Z.; Visualization: X.M.Q. and M.Y.X.; Original draft, Review \& editing: X.M.Q., S.L., L.L. and Z.Y.Z;; All authors have read and approved the final manuscript.
Funding

This study was funded by Jiangsu Provincial Medical Youth Talent (No. QNRC2016040). The funding source had no role in the study design, data collection, data analysis, or interpretation of the findings.

\section{Availability of data and materials}

The datasets used and analyzed during the current study are available from the corresponding author on reasonable request.

\section{Declarations}

Ethics approval and consent to participate

This retrospective study was approved by the Ethical Committee of Nanjing Drum Tower Hospital, The Affiliated Hospital of Nanjing University Medical School (Approval Documents Number: 2020-032-01). The requirement for informed consent was waived.

\section{Consent for publication}

Not applicable.

\section{Competing interests}

The authors declare that they have no competing interests.

Received: 9 March 2021 Accepted: 9 August 2021

Published online: 16 September 2021

\section{References}

1. Bray F, Ferlay J, Soerjomataram I, Siegel RL, Torre LA, Jemal A. Global cancer statistics 2018: GLOBOCAN estimates of incidence and mortality worldwide for 36 cancers in 185 countries. CA Cancer J Clin. 2018;68(6):394-424. https://doi.org/10.3322/caac.21492.

2. Kim JM, Jung $\mathrm{H}$, Lee JS, Lee $\mathrm{HH}$, Song $\mathrm{KY}$, Park $\mathrm{CH}$, et al. Clinical implication of serosal change in pathologic subserosa-limited GC. World I Surg. 2012; 36(2):355-61. https://doi.org/10.1007/s00268-011-1334-X.

3. Tokumitsu Y, Yoshino S, lida M, Yoshimura K, Ueno T, Hazama S, et al. Intraoperative dissemination during gastrectomy for gastric cancer associated with serosal invasion. Surg Today. 2015;45(6):746-51. https://doi. org/10.1007/s00595-014-1005-2.

4. Peng $Y F$, Imano $M$, Itoh $T$, Satoh $T$, Chiba $Y$, Imamoto $H$, et al. A phase II trial of perioperative chemotherapy involving a single intraperitoneal administration of paclitaxel followed by sequential S-1 plus intravenous paclitaxel for serosa-positive gastric cancer. J Surg Oncol. 2015;111(8):10416. https://doi.org/10.1002/jso.23928.

5. Ajani JA, D'Amico TA, Almhanna K, et al. National Comprehensive Cancer Network. NCCN clinical practice guidelines in oncology. Gastric Cancer. Version 1.2019. Available at: https://www.nccn.org/professionals/physician_ gls/PDF/gastric.pdf. Accessed 14 May 2019.

6. Edge SB, Byrd DR, Compton CC, et al., editors. AJCC cancer staging manual. 7th ed. New York: Springer; 2010.

7. Amin $\mathrm{MB}$, Edge $\mathrm{SB}$, Greene $\mathrm{FL}$, et al., editors. AJCC Cancer staging manual. 8th ed. New York: Springer; 2017. https://doi.org/10.1007/978-3-319-40618-3.

8. Kim DJ, Lee JH, Kim W. Impact of intraoperative macroscopic diagnosis of serosal invasion in pathological subserosal (pT3) GC. J Gastric Cancer. 2014; 14(4):252-8. https://doi.org/10.5230/jgc.2014.14.4.252.

9. Lee SL, Ku YM, Jeon HM, Lee HH. Impact of the cross-sectional location of multidetector computed tomography scans on prediction of serosal exposure in patients with advanced gastric cancer. Ann Surg Oncol. 2017; 24(4):1003-9. https://doi.org/10.1245/s10434-016-5670-9.

10. Kim TU, Kim S, Lee JW, Lee NK, Jeon TY, Park DY. MDCT features in the differentiation of T4a GC from less-advanced GC: significance of the hyperattenuating serosa sign. Br J Radiol. 2013;86(1029):20130290. https:// doi.org/10.1259/bjr.20130290. 
11. Cardoso R, Coburn N, Seevaratnam R, et al. A systematic review and metaanalysis of the utility of EUS for preoperative staging for gastric cancer. Gastric Cancer. 2012;15(Suppl 1):S19-26.

12. Seevaratnam R, Cardoso R, McGregor C, et al. How useful is preoperative imaging for tumor, node, metastasis (TNM) staging of gastric cancer? A meta-analysis. Gastric Cancer. 2012;15(Suppl 1):S3-S18.

13. Sun RJ, Fang MJ, Tang L, Li XT, Lu QY, Dong D, et al. CT-based deep learning radiomics analysis for evaluation of serosa invasion in advanced gastric cancer. Eur J Radiol. 2020;132:109277. https://doi.org/10.1016/j.ejrad.2 020.109277.

14. Wang Y, Liu W, Yu Y, Liu JJ, Xue HD, Qi YF, et al. CT radiomics nomogram for the preoperative prediction of lymph node metastasis in GC. Eur Radiol. 2020;30(2):976-86. https://doi.org/10.1007/s00330-019-06398-z.

15. Liu S, He J, Liu S, Ji C, Guan W, Chen L, et al. Radiomics analysis using contrast-enhanced $\mathrm{CT}$ for preoperative prediction of occult peritoneal metastasis in advanced GC. Eur Radiol. 2020;30(1):239-46. https://doi.org/1 0.1007/s00330-019-06368-5

16. Li Q, Qi L, Feng QX, Liu C, Sun SW, Zhang J, et al. Machine learning-based computational models derived from large-scale radiographic-Radiomic images can help predict adverse histopathological status of gastric cancer Clin Transl Gastroenterol. 2019;10(10):e00079. https://doi.org/10.14309/ctg. 0000000000000079

17. Li W, Zhang L, Tian C, Song H, Fang M, Hu C, et al. Prognostic value of computed tomography radiomics features in patients with GC following curative resection. Eur Radiol. 2019;29(6):3079-89. https://doi.org/10.1007/ s00330-018-5861-9.

18. Ganeshan B, Miles KA. Quantifying tumour heterogeneity with CT. Cancer Imaging. 2013;13(1):140-9. https://doi.org/10.1102/1470-7330.2013.0015.

19. Liu S, Shi H, Ji C, et al. Preoperative CT texture analysis of gastric cancer: correlations with postoperative TNM staging. Clin Radiol. 2018; 73(8):756.e1-9.

20. Wang $Y$, Liu W, Yu Y, Liu JJ, Jiang L, Xue HD, et al. Prediction of the depth of tumor invasion in gastric cancer: potential role of CT Radiomics. Acad Radiol. 2020;27(8):1077-84. https://doi.org/10.1016/j.acra.2019.10.020.

21. Liu PS, Platt JF. CT angiography in the abdomen: a pictorial review and update. Abdom Imaging. 2014;39(1):196-214. https://doi.org/10.1007/s002 61-013-0035-3.

22. Waldum HL, Fossmark R. Types of gastric carcinomas. Int J Mol Sci. 2018; 19(12):4109. https://doi.org/10.3390/ijms19124109.

23. Lee IJ, Lee JM, Kim SH, Shin Cll, Lee JY, Kim SH, et al. Diagnostic performance of 64-channel multidetector $C T$ in the evaluation of gastric cancer: differentiation of mucosal cancer (T1a) from submucosal involvement (T1b and T2). Radiology. 2010;255(3):805-14. https://doi.org/1 $0.1148 /$ radiol.10091313.

24. Ma Z, Liang C, Huang Y, He L, Liang C, Chen X, et al. Can lymphovascular invasion be predicted by preoperative multiphasic dynamic $C T$ in patients with advanced gastric cancer? Eur Radiol. 2017;27(8):3383-91. https://doi. org/10.1007/s00330-016-4695-6.

25. Liu S, Qiao X, Xu M, Ji C, Li L, Zhou Z. Development and validation of multivariate models integrating preoperative clinicopathological parameters and radiographic findings based on late arterial phase ct images for predicting lymph node metastasis in gastric cancer. Acad Radiol. 2021; S1076-6332(21)00020-9. [published online ahead of print, 2021 Jan 21].

26. Dong D, Tang L, Li ZY, et al. Development and validation of an individualized nomogram to identify occult peritoneal metastasis in patients with advanced gastric cancer. Ann Oncol. 2019;30(3):431-8. https://doi.org/1 0.1093/annonc/mdz001.

27. Fukayama M, Rugge M, Washington MK. Tumors of the stomach. In: WHO classification of tumours editorial board. Digestive system tumours WHO classification of tumours. 5th ed. Lyon: IARC; 2019.

28. Dong D, Fang MJ, Tang L, Shan XH, Gao JB, Giganti F, et al. Deep learning radiomic nomogram can predict the number of lymph node metastasis in locally advanced gastric cancer: an international multicenter study. Ann Oncol. 2020;31(7):912-20. https://doi.org/10.1016/j.annonc.2020.04.003.

29. Duffy MJ, Lamerz R, Haglund C, Nicolini A, Kalousová M, Holubec L, et al. Tumor markers in colorectal cancer, gastric cancer and gastrointestinal stromal cancers: European group on tumor markers 2014 guidelines update. Int J Cancer. 2014;134(11):2513-22. https://doi.org/10.1002/ijc.28384.

30. Tian SB, Yu JC, Kang WM, Ma ZQ, Ye X, Cao ZJ, et al. Combined detection of CEA, CA 19-9, CA 242 and CA 50 in the diagnosis and prognosis of resectable gastric cancer. Asian Pac J Cancer Prev. 2014;15(15):6295-300 https://doi.org/10.7314/APJCP.2014.15.15.6295.

31. Kim DK, Kang SH, Kim JS, Rou WS, Joo JS, Kim MH, et al. Feasibility of using two-dimensional axial computed tomography in pretreatment decision making for patients with early gastric cancer. Medicine (Baltimore). 2020; 99(4):e18928. https://doi.org/10.1097/MD.0000000000018928.

32. Lee SY, Ju MK, Jeon HM, et al. Regulation of tumor progression by programmed necrosis. Oxidative Med Cell Longev. 2018;2018:3537471.

33. Maynard J, Okuchi S, Wastling S, Busaidi AA, Almossawi O, Mbatha W, et al. World Health Organization grade II/II glioma molecular status: prediction by MRI morphologic features and apparent diffusion coefficient. Radiology. 2020;296(1):111-21. https://doi.org/10.1148/radiol.2020191832.

34. Yang L, Yang J, Zhou X, Huang L, Zhao W, Wang T, et al. Development of a radiomics nomogram based on the $2 \mathrm{D}$ and $3 \mathrm{D} C \mathrm{CT}$ features to predict the survival of non-small cell lung cancer patients. Eur Radiol. 2019;29(5):2196206. https://doi.org/10.1007/s00330-018-5770-y.

35. You MW, Park S, Kang HJ, Lee DH. Radiologic serosal invasion sign as a new criterion of T4a GC on computed tomography: diagnostic performance and prognostic significance in patients with advanced GC. Abdom Radiol (NY). 2020;45(10):2950-9. https://doi.org/10.1007/s00261-019-02156-3.

\section{Publisher's Note}

Springer Nature remains neutral with regard to jurisdictional claims in published maps and institutional affiliations.
Ready to submit your research? Choose BMC and benefit from:

- fast, convenient online submission

- thorough peer review by experienced researchers in your field

- rapid publication on acceptance

- support for research data, including large and complex data types

- gold Open Access which fosters wider collaboration and increased citations

- maximum visibility for your research: over $100 \mathrm{M}$ website views per year

At BMC, research is always in progress.

Learn more biomedcentral.com/submissions 